\title{
Grand Challenge in Viral Disease Investigation: An Endless Endeavor
}

\begin{abstract}
Javier Buesa ${ }^{1,2 *}$
${ }^{1}$ Department of Microbiology, School of Medicine, University of Valencia, Valencia, Spain, ${ }^{2}$ Microbiology Service, Clinic University Hospital, Instituto de Investigación Sanitaria del Hospital Clínico de Valencia Health Research Institute, Valencia, Spain
\end{abstract}

Keywords: viral infection, viral disease, susceptibility, symptoms, immune response, immunosuppression, epidemic, oncogenesis

Emerged viral diseases causing pandemics (e.g., AIDS, $2009 \mathrm{H} 1 \mathrm{~N} 1$ pandemic flu, COVID-19) are of major concern for human health. Viral diseases were known long before the discovery of viruses, but the history of Virology is quite recent, and we know that it will never end up. Viral diseases constitute a permanent defiance for humans, as well as for animals, plants, fungi, protozoa, and bacteria. Viruses are continuously evolving and this fact has a direct impact on their pathogenic potential. The outcome of viral infections can be extraordinarily diverse, from asymptomatic infections to disease and death.

Viruses can disturb the normal physiological parameters of an organism by many different mechanisms. They can perturb the homeostasis and induce immunopathology, immunosuppression, cellular transformation, oncogenesis, and direct cell death. Viral pathogenesis has been regarded as a phenomenological discipline with many observations, but scarce mechanistic insights.

Some viruses can escort us all our life long (e.g., herpesviruses, polyomaviruses). They live within our own bodies, making up our virome. This includes viruses that cause acute, chronic, latent or persistent infections, viruses infecting bacteria, archaea, and endogenous viral elements integrated into host chromosomes. Some viruses are nonpathogenic and others are extremely virulent and lethal. The reasons why some viruses are virulent and others are not remain elusive and can change from one virus to another. It is feasible to reduce viral virulence through mutagenesis, but this does not demonstrate why some viruses are virulent. In addition, viruses can change their relationship with the hosts and modify their pathogenic pattern.

A great deal of our knowledge about viruses, immunologic tolerance, and immunopathology came from studies on lymphocytic choriomeningitis virus (LCMV) infection in mouse, its natural host (1). Animal models have been extremely useful to investigate viral-host relationships, how viruses cause disease, and the mechanisms of the immune response. It is convenient to recall the discoveries of MHC-restricted cytotoxic T lymphocyte function (2), the mechanisms involved in cytotoxic T cell memory (3), or T lymphocyte exhaustion (4).

\section{HUMAN VIROME}

Deep sequencing technologies are modifying our previous ideas about viruses. We can now investigate thousands of viruses that inhabit our bodies without causing any clinical symptoms. By studying their interactions with our cells, host genetics, and the immune system, we can analyze their effects on health and disease. An example of nonpathogenic and ubiquitous viruses is Torque Teno virus (TTV), a small DNA-virus of the genus Alphatorquevirus in the Anelloviridae family, whose replication is linked to the immune status of the host. TTVs can cause some level of viremia in immunocompetent individuals (5) but no association of TTV to any human disease has been found so far (6). As TTV is associated with immunosuppression, it is being applied to control the degree of immunosuppression in patients after organ transplantation (7). This is a beneficial use of a virus present in our virome, an area that deserves further research. 


\section{PATTERNS OF VIRAL DISEASES}

The type of disease that viruses can cause is determined by their cellular tropism, the host response, and the course of the infection-acute, chronic, persistent, latent, reactivation, slow infection, transforming, or oncogenic. Acute infections occur when host defenses are modulated within a short time. After innate immunity activation, viruses will be cleared by the adaptive immune response. Chronic and persistent infections result when the innate and adaptive immune systems are essentially ineffective for a long period of time. For many noncytolytic viruses, immunopathology is the basis of disease (8). Consequently, viral disease investigation has to be accomplished with a multidisciplinary perspective, involving molecular virology, pathology, immunology, genetics, preclinical, and clinical studies, as well as epidemiology. Each viral species and even each viral type, variant or strain, may represent a different scenario in terms of pathophysiology, innate, and adaptive immune responses and clinical outcome.

\section{OLD AND EMERGING VIRUSES CAUSING NOVEL DISEASES}

Signs and symptoms, the clinical features associated to infectious diseases, are usually well-known, although they can shift slowly and also abruptly.

A high incidence of cases of acute flaccid myelitis was coincidental in different countries in 2014 and 2016 with an increase in the circulation of enterovirus D68 (EV-D68) strains (9). The EV-D68 emergence showed many similarities with poliovirus a century ago, suggesting that this enterovirus could be considered the culprit of a "new polio" (10). Applying the Bradford Hill criteria to investigate a causal relationship it could be establish an etiological role of EV-D68 to acute flaccid myelitis (9). Fredricks and Relman (11) and Lipkin (12) emphasized the relevance of molecular diagnostics and the power of epidemiological analyses to consider the etiological role of pathogens, especially viruses, in diseases. In order to improve the diagnosis of enterovirus infections and establish the surveillance of enterovirus types, a European non-polio enterovirus network (ENPEN) was established. The first goal of that international network was to facilitate a rapid and accurate diagnosis of enterovirus infections (13).

In a similar manner, enterovirus A71 (EV-A71), regarded an emerging pathogen, has been associated with a wide range of clinical disorders including severe neurological syndromes. In the past 20 years, this virus has been linked with large outbreaks of hand, foot, and mouth disease with neurological complications in the Asia-Pacific region. In spring 2016, EV-A71 was found to be the etiological agent of an outbreak of severe neurological illness, including brainstem encephalitis $(14,15)$. The analysis of intrathecal antiviral antibodies using a pan-viral phage display library (VirScan) implicated non-polio enteroviruses in acute flaccid paralysis (16).

Another example of new viruses causing unexpected diseases are the novel astroviruses which were discovered in 2008-09 in human stool and were associated with severe cases of meningitis and encephalitis (17).

High-throughput sequencing is a powerful tool to discover new viral pathogens associated to novel or old diseases without a defined causative agent. The application of this technology during an outbreak of fatal disease in transplant patients allowed the identification of a new arenavirus that had been transmitted through solid-organ transplantation (18). Among the most remarkable and striking novel viral diseases, congenital Zika virus disease stands out. Zika virus was discovered in 1952 (19) but it had not aroused much interest, until epidemics emerged in Central and South America between 2013 and 2016, with serious congenital malformations consisting of severe microcephaly and neurological complications in adults (20). In contrast to other arboviruses, Zika virus can be spread by mosquito vectors but also by sexual and maternal-to-fetal transmission (20-22).

The emergence of COVID-19 forced to a throughout analysis of the clinical symptoms displayed by this new viral disease (23). It was reported, for instance, that anosmia and ageusia are major symptoms in a high number of patients (24). The pathophysiology of symptoms in mild and severe SARSCoV-2 infections, and in post-COVID-19 syndrome, is still being investigated.

Recently, a new orthonairovirus, named Songling virus, was identified in the Chinese province of Heiglongjiang from patients with headache, fever, fatigue, and depression, who had been previously bitten by ticks (25). The conclusion to be drawn is that the etiology of new syndromes that arise as outbreaks should always be explored.

\section{SUSCEPTIBILITY TO VIRAL INFECTION AND DISEASE}

Susceptibility to different viruses and disease progression are highly variable, and depends on many factors: age, nutritional status, pre-existing chronic diseases, minimal infectious dose, site of entry, immunity, and genetic background of the individual. Host genetic polymorphism determines susceptibility to viral infections. It is known that MHC polymorphism increases resistance to disease in humans (26). A selective advantage was obtained from that diversity and influenced human evolution. For instance, a polymorphism in the chemokine receptor CCR5, co-receptor for HIV-1, prevents the virus from entering the cell, conferring resistance to infection (27). In principle, polymorphism in any genes encoding host proteins involved in the replicacion cycle of a virus could intervene in the susceptibility to that specific virus.

Some viral diseases are highly contagious and others show a very low case-to-infection rate. For measles, the basic reproduction number $\mathrm{R}_{0}$ is considered to be $12-18$, meaning that each infected person with measles virus would, on average, spread the disease to $12-18$ other people in a fully susceptible population (28). $R_{0}$ is useful to estimate the herd immunity threshold and the vaccination coverage necessary to control the transmission of an infectious disease. 
The knowledge of the interactions of enteric viruses with glycoconjugates in the intestinal mucosa has encouraged the study of their glycobiology. Noroviruses and rotaviruses are the leading agents causing viral gastroenteritis worldwide. Susceptibility to these viruses has been associated with human histo-blood group antigens (HBGAs) present on the intestinal mucosa, which are associated with infection caused by several viral genotypes (29-32). These studies are relevant, as the dissection of the interactions between viruses and their hosts is relevant to develop effective antiviral strategies.

\section{PATHOPHYSIOLOGY OF VIRAL DISEASES: PRE-CLINICAL AND CLINICAL RESEARCH}

Many clinical research studies aim to clarify how viruses do cause disease. For this purpose, pre-clinical experimental models are indispensable tools for studying the biology of virus infection, and they have provided important insight of the cellular factors and biological mechanisms involved in the establishment of acute viral infection, persistence, and carcinogenesis.

In many viral diseases there is an imbalance of the innate immune response that contributes to clinical outcome (33). Type I interferon (IFN) deficiency is considered a feature of severe COVID-19. SARS-CoV-2 infects human bronchial epithelial cells displaying a limited IFN response (34). It has been reported that patients with mild COVID-19 show elevated interferon-stimulated genes (ISGs) expression, which is absent in patients with severe disease (35). Some COVID-19 patients suffer a sudden worsening with the onset of the cytokine storm syndrome. The host immune response to the virus in those cases is hyperactive, with an excessive production of inflammatory cytokines, including IL-1, TNF- $\alpha$, and IL-6 (36). Regarding COVID-19 pathophysiology, acute respiratory distress syndrome, cytokine storm, exhausted $\mathrm{T}$ lymphocytes, and lymphopenia are the main proposed mechanisms in the pathogenesis and immunopathology of the disease $(37,38)$.

In the last years, it is becoming more evident that an interaction between infecting viruses and microbiota may take place, influencing the course of the disease. As an example, influenza patients often suffer of diarrhea, but the pathophysiological mechanism is not well-understood. It has been experimentally shown that the virus induces intestinal immune inflammation via microbiota-mediated Th17 cells, which can explain why flu patients develop intestinal symptoms (39). It has also been demonstrated that host genetic factors like the histo-blood group antigens (HBGA) are associated with susceptibility to enteric virus infections. Recent studies point to HBGAs as factors that modulate the intestinal microbiota, which can additionally influence on the viral infection outcome $(29,40)$.

\section{EPIDEMICS AND PANDEMICS}

Acute viral infections can cause serious epidemics affecting millions of individuals every year (e.g., influenza virus, dengue virus, Zika virus, and currently SARS-CoV-2).
The current pandemic of COVID-19 has created a global health crisis, disrupting social life and economy all over the world. This pandemic is by no means the first event in its kind for humanity, as there are records of the huge impact that H1N1 influenza pandemic had in 1918-19, which was a global health catastrophe and killed more than 50 million people worldwide (41). Lessons have to be learned regarding a better manage of epidemiological assessment, public health policies, prevention strategies, and potential therapeutic interventions for viral pandemics in the future.

\section{VIRAL ONCOGENESIS}

Some viruses are involved in the genesis of benign and malignant tumors. The first DNA virus found to be oncogenic was a rabbit papilloma virus discovered in 1933 (42). In human viral oncogenesis, host genetics, and the fact that viruses contribute partially to the oncogenic events reflects the multistep nature of the process (43). Oncogenic viruses are members of different families that share some general features. Cells transformed by oncogenic viruses generally retain viral DNA in their nuclei, integrated into cellular chromosomes or as episomes. There are two classes of viral oncogenes based on their similarity to cellular sequences. Oncogenes of transducing retroviruses and certain herpesviruses, like HHV-8, the etiologic agent of Kaposi's sarcoma, are closely related to cellular genes. Viral oncogenes in the second class, such as adenovirus E1A and polyomavirus LT, do not seem to be related to cellular genes and their origin remains a conundrum (44). Proteins encoded by transforming genes of both RNA and DNA viruses can alter cellular signal transduction cascades, with activation of pathways that stimulate cellular proliferation and cancer. Some tumors of viral etiology can be prevented via vaccination (e.g., HBV and HPV types 16 and 18) and viral oncogenesis remains consequently a major topic of research.

\section{VIRUSES AND IMMUNOSUPPRESSION}

Virus-induced immunosuppression can range from a slight attenuation of the immune response to a severe global inhibition. HIV-1 is known to cause severe immunodeficiency, but other viruses can also cause it, such as measles and rubella. The high mortality caused by measles virus, responsible for more than 100,000 deaths annually, is due to opportunistic infections resulting from immunosuppression induced by this virus (45, 46). Some viruses can suppress effective responses to their own antigens and promote the development of persistent infections. It was experimentally demonstrated that immune system-mediated destruction of virus-infected dendritic cells results in generalized immune suppression, contributing to the clinically important immunosuppression associated with many virus infections (47). Nevertheless, it is still important to elucidate all the mechanisms involved in clinically important virus-induced suppression of the host immune response. 


\section{ANTIVIRAL CHEMOTHERAPY}

Treatment of viral diseases is still a major challenge. Viruses do not have affordable targets to aim as it happens with bacteria, which are prokaryotic cells with different molecular components that can be inactivated without harming our own cells. Virusinfected cells harbor invaders that control their biosynthetic machinery, and need to be eliminated, cured, or restored. The current antiviral repertoire includes many drugs, but effective therapies for many viral infections are still needed (48).

\section{REFERENCES}

1. Oldstone MBA. Lessons learned and concepts formed from study of the pathogenesis of the two negative-strand viruses lymphocytic choriomeningitis and influenza. Proc Natl Acad Sci USA. (2013) 110:4180-3. doi: 10.1073/pnas.1222025110

2. Doherty PC, Zinkernagel RM. H-2 compatibility is required for T cell mediated lysis of target cells infected with lymphocytic choriomeningitis virus. J Exp Med. (1975) 141:502-7. doi: 10.1084/jem.141.2.502

3. Lau LL, Jamieson BD, Somasundaram T, Ahmed R. Cytotoxic T-cell memory without antigen. Nature. (1994) 369:648-52. doi: 10.1038/369648a0

4. Angelosanto JM, Blackburn SD, Crawford A, Wherry EJ. Progressive loss of memory $\mathrm{T}$ cell potential and commitment to exhaustion during chronic viral infection. J Virol. (2012) 86:8161-70. doi: 10.1128/JVI.00889-12

5. Haloschan M, Bettesch R, Görzer I, Weseslindtner L, Kundi M, PuchhammerStöckl E. TTV DNA plasma load and its association with age, gender, and HCMV IgG serostatus in healthy adults. Age (Omaha). (2014) 36:9716. doi: 10.1007/s11357-014-9716-2

6. Kincaid RP, Burke JM, Cox JC, de Villiers EM, Sullivan CS. A human Torque Teno virus encodes a microRNA that inhibits interferon signaling. PLoS Pathog. (2013) 9:e1003818. doi: 10.1371/journal.ppat.1003818

7. Jaksch P, Kundi M, Görzer I, Muraközy G, Lambers C, Benazzo A, et al. Torque teno virus as a novel biomarker targeting the efficacy of immunosuppression after lung transplantation. J Infect Dis. (2018) 218:19228. doi: 10.1093/infdis/jiy452

8. Keeling MJ, Rohani P. Modeling Infectious Diseases in Humans and Animals. Princeton, NJ: Princeton University Press (2011).

9. Messacar K, Asturias EJ, Hixon AM, Van Leer-Buter C, Niesters HGM, Tyler KL, et al. Enterovirus D68 and acute flaccid myelitisevaluating the evidence for causality. Lancet Infect Dis. (2018) 18:e239. doi: 10.1016/S1473-3099(18)30094-X

10. Cassidy H, Poelman R, Knoester M, Leer-Buter CCV, Niesters HGM. Enterovirus D68 -the new polio? Front Microbiol. (2018) 9:2677. doi: 10.3389/fmicb.2018.02677

11. Fredricks DN, Relman DA. Sequence-based identification of microbial pathogens: a reconsideration of Koch's postulates. Clin Microbiol Rev. (1996) 9:18-33. doi: 10.1128/CMR.9.1.18

12. Lipkin WI. Pathogen discovery. PLoS Pathog. (2008) 4:e1000002. doi: 10.1371/journal.ppat.1000002

13. Harvala $\mathrm{H}$, Broberg E, Benschop $\mathrm{K}$, Berginc $\mathrm{N}$, Ladhani $\mathrm{S}$, Susi P, et al. Recommendations for enterovirus diagnostics and characterisation within and beyond Europe. J Clin Virol. (2018) 101:11-17. doi: 10.1016/j.jcv.2018.01.008

14. González-Sanz R, Casas-Alba D, Launes C, Muñoz-Almagro C, Montserrat Ruiz-García M, Alonso $\mathrm{M}$, et al. Molecular epidemiology of an enterovirus A71 outbreak associated with severe neurological disease, Spain, 2016. Eurosurveillance. (2019) 24:1800089. doi: 10.2807/1560-7917.ES.2019.24.7.1800089

15. Leon KE, Schubert RD, Casas-Alba D, Hawes IA, Ramachandran PS, Ramesh A, et al. Genomic and serologic characterization of enterovirus A71 brainstem encephalitis. Neurol Neuroimmunol Neuroinflammation. (2020) 7:e703. doi: 10.1212/NXI.0000000000000703
Viral disease investigation remains a major challenge for clinicians, basic and clinical virologists, immunologists, geneticists, pathologists, pharmacologists, epidemiologists, and public health specialists.

\section{AUTHOR CONTRIBUTIONS}

The author confirms being the sole contributor of this work and has approved it for publication.

16. Schubert RD, Hawes IA, Ramachandran PS, Ramesh A, Crawford ED, Pak JE, et al. Pan-viral serology implicates enteroviruses in acute flaccid myelitis. Nat Med. (2019) 25:1748-52. doi: 10.1038/s41591-019-0613-1

17. Vu DL, Cordey S, Brito F, Kaiser L. Novel human astroviruses: novel human diseases? J Clin Virol. (2016) 82:56-63. doi: 10.1016/j.jcv.2016.07.004

18. Palacios G, Druce J, Du L, Tran T, Birch C, Briese T, et al. A new arenavirus in a cluster of fatal transplant-associated diseases. N Engl J Med. (2008) 358:991-8. doi: 10.1056/NEJMoa073785

19. Dick, G. W.A., Kitchen SF, Haddow AJ. Zika Virus (I). Isolations and serological specificity. Trans R Soc Trop Med Hyg. (1952) 46:50920. doi: 10.1016/0035-9203(52)90042-4

20. Lee CYP, Ng LFP. Zika virus: from an obscurity to a priority. Microbes Infect. (2018) 20:635-45. doi: 10.1016/j.micinf.2018.02.009

21. Musso D, Roche C, Robin E, Nhan T, Teissier A, Cao-Lormeau VM. Potential sexual transmission of Zika virus. Emerg Infect Dis. (2015) 21:35961. doi: 10.3201/eid2102.141363

22. Besnard M, Lastère S, Teissier A, Cao-Lormeau VM, Musso D. Evidence of perinatal transmission of Zika virus, French Polynesia, December 2013 and February 2014. Eurosurveillance. (2014) 19:20751. doi: 10.2807/1560-7917.ES2014.19.13.20751

23. Pascarella G, Strumia A, Piliego C, Bruno F, Del Buono R, Costa F, et al. COVID-19 diagnosis and management: a comprehensive review. J Intern Med. (2020) 288:192-6. doi: 10.1111/joim.13091

24. Vaira LA, Salzano G, Deiana G, De Riu G. Anosmia and ageusia: common findings in COVID-19 patients. Laryngoscope. (2020) 130:178. doi: 10.1002/lary.28837

25. Ma J, Lv XL, Zhang X, Han SZ, Wang ZD, Li L, et al. Identification of a new orthonairovirus associated with human febrile illness in China. Nat Med. (2021) 27:434-9. doi: 10.1038/s41591-020-01228-y

26. Hughes AL, Nei M. Maintenance of MHC polymorphism. Nature. (1992) 355:402-3. doi: 10.1038/355402b0

27. Liu R, Paxton WA, Choe S, Ceradini D, Martin SR, Horuk R, et al. Homozygous defect in HIV-1 coreceptor accounts for resistance of some multiply-exposed individuals to HIV-1 infection. Cell. (1996) 86:36777. doi: 10.1016/S0092-8674(00)80110-5

28. Guerra FM, Bolotin S, Lim G, Heffernan J, Deeks SL, Li Y, et al. The basic reproduction number (R0) of measles: a systematic review. Lancet Infect Dis. (2017) 17:e420-e8. doi: 10.1016/S1473-3099(17)30307-9

29. Rodríguez-Díaz J, García-Mantrana I, Vila-Vicent S, Gozalbo-Rovira R, Buesa J, Monedero V, et al. Relevance of secretor status genotype and microbiota composition in susceptibility to rotavirus and norovirus infections in humans. Sci Rep. (2017) 7:45559. doi: 10.1038/srep45559

30. Nordgren J, Svensson L. Genetic susceptibility to human norovirus infection: an update. Viruses. (2019) 11:226. doi: 10.3390/v110 30226

31. Le Pendu J, Ruvoën-Clouet N, Kindberg E, Svensson L. Mendelian resistance to human norovirus infections. Semin Immunol. (2006) 18:37586. doi: 10.1016/j.smim.2006.07.009

32. Gozalbo-Rovira R, Ciges-Tomas JR, Vila-Vicent S, Buesa J, Santiso-Bellón C, Monedero V, et al. Unraveling the role of the secretor antigen in human rotavirus attachment to histo-blood group antigens. PLoS Pathog. (2019) 15:e1007865. doi: 10.1371/journal.ppat.1007865 
33. Chan CYY, Chan KR, Chua CJH, nur Hazirah S, Ghosh S, Ooi EE, et al. Early molecular correlates of adverse events following yellow fever vaccination. JCI Insight. (2017) 2:e96031 doi: 10.1172/jci.insight.96031

34. Zhang Y, Gargan S, Lu Y, Stevenson NJ. An overview of current knowledge of deadly CoVs and their interface with innate immunity. Viruses. (2021) 13:1-33. doi: 10.3390/v13040560

35. Bastard P, Michailidis E, Hoffmann HH, Chbihi M, Le Voyer T, Rosain J, et al. Auto-antibodies to type I IFNs can underlie adverse reactions to yellow fever live attenuated vaccine. J Exp Med. (2021) 218:e20202486. doi: 10.1084/jem.20202486

36. Blanco-Melo D, Nilsson-Payant BE, Liu WC, Uhl S, Hoagland D, Møller $\mathrm{R}$, et al. Imbalanced host response to SARS-CoV-2 drives development of COVID-19. Cell. (2020) 181:1036-45.e9. doi: 10.1016/j.cell.2020.04.026

37. Wauters E, Thevissen K, Wouters C, Bosisio FM, De Smet F, Gunst $\mathrm{J}$, et al. Establishing a unified COVID-19 "Immunome": Integrating coronavirus pathogenesis and host immunopathology. Front Immunol. (2020) 11:1642. doi: $10.3389 /$ fimmu.2020.01642

38. Vardhana SA, Wolchok JD. The many faces of the anti-COVID immune response. J Exp Med. (2020) 217:e20200678. doi: 10.1084/jem.20200678

39. Wang J, Li F, Wei H, Lian ZX, Sun R, Tian Z. Respiratory influenza virus infection induces intestinal immune injury via microbiotamediated Th17 cell-dependent inflammation. J Exp Med. (2014) 211:2397-410. doi: 10.1084/jem.20140625

40. Monedero V, Buesa J, Rodríguez-Díaz J. The interactions between host glycobiology, bacterial microbiota, and viruses in the gut. Viruses. (2018) 10:96. doi: 10.3390/v10020096

41. Martini M, Gazzaniga V, Bragazzi NL, Barberis I. The Spanish Influenza Pandemic: a lesson from history 100 years after 1918. J Prev Med Hyg. (2019) 60:E64-E7. doi: 10.15167/2421-4248/jpmh2019.60.1.1205

42. Shope RE, Hurst EW. Infectious papillomatosis of rabbits. J Exp Med. (1933) 58:607-24. doi: 10.1084/jem.58.5.607
43. Mesri EA, Feitelson MA, Munger K. Human viral oncogenesis: a cancer hallmarks analysis. Cell Host Microbe. (2014) 15:26682. doi: 10.1016/j.chom.2014.02.011

44. Huh K, Zhou X, Hayakawa H, Cho J-Y, Libermann TA, Jin J, et al. Human Papillomavirus type 16 E7 oncoprotein associates with the cullin 2 ubiquitin ligase complex, which contributes to degradation of the retinoblastoma tumor suppressor. J Virol. (2007) 81:9737-47. doi: 10.1128/JVI. 00881-07

45. Mina MJ, Kula T, Leng Y, Li M, De Vries RD, Knip M, et al. Measles virus infection diminishes preexisting antibodies that offer protection from other pathogens. Science. (2019) 366:599-606. doi: 10.1126/science. aay6485

46. Du Toit A. Measles increases the risk of other infections. Nat Rev Microbiol. (2020) 18:2. doi: 10.1038/s41579-019-0301-7

47. Borrow P, Evans CF, Oldstone MB. Virus-induced immunosuppression: immune system-mediated destruction of virus-infected dendritic cells results in generalized immune suppression. J Virol. (1995) 69:105970. doi: 10.1128/JVI.69.2.1059-1070.1995

48. De Clercq E, Li G. Approved antiviral drugs over the past 50 years. Clin Microbiol Rev. (2016) 29:695-747. doi: 10.1128/CMR.00102-15

Conflict of Interest: The author declares that the research was conducted in the absence of any commercial or financial relationships that could be construed as a potential conflict of interest.

Copyright (C) 2021 Buesa. This is an open-access article distributed under the terms of the Creative Commons Attribution License (CC BY). The use, distribution or reproduction in other forums is permitted, provided the original author(s) and the copyright owner(s) are credited and that the original publication in this journal is cited, in accordance with accepted academic practice. No use, distribution or reproduction is permitted which does not comply with these terms. 